\title{
Electrochemically Active, Compressible, and Conducting Silk Fibroin Hydrogels
}

\author{
Jiaojiao Yang, Jun Luo, Haidong Liu, Liyang Shi, Ken Welch, Zhaohui Wang,* and Maria Strømme
}

Cite This: Ind. Eng. Chem. Res. 2020, 59, 9310-9317

Read Online
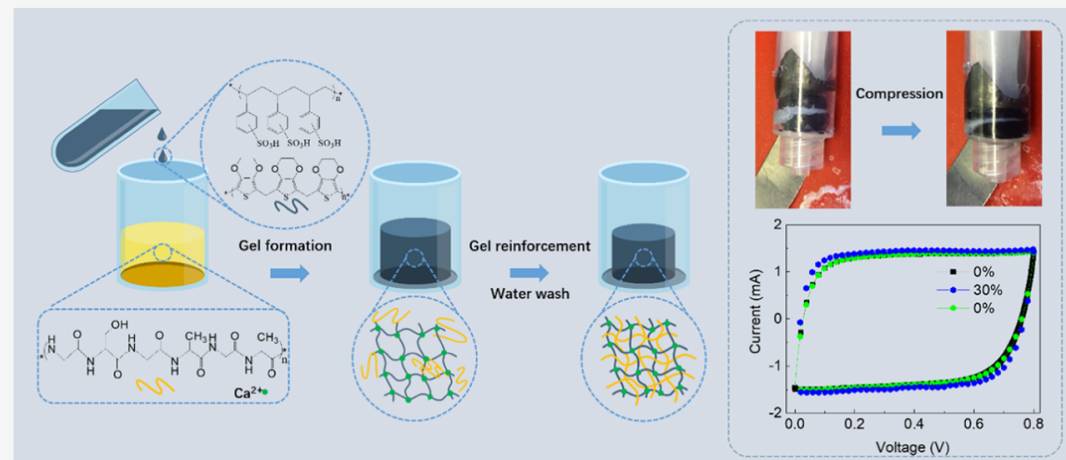

ABSTRACT: Silk fibroin-based conducting hydrogels possess hierarchical structural motifs featuring unique properties, but the development of such materials has proven to be challenging. Herein, we develop a novel strategy for the fabrication of a conducting silk fibroin hydrogel based on an interpenetrated network of poly(3,4-ethylene dioxythiophene):poly(styrene sulfonate) (PEDOT:PSS) and silk fibroin. The hydrogel possesses good electrical conductivity and considerable capacitance and cycling stability due to the existence of the PEDOT conducting network, as well as enhanced mechanical properties such as compressibility due to $\beta$-sheets in the silk fibroin network and $\mathrm{Ca}^{2+}$ cross-linking of the PSS components. A symmetric charge storage device based on conductive silk fibroin hydrogel electrodes exhibited a remarkable areal capacitance of $1.1 \mathrm{~F} \mathrm{~cm}^{-2}$ at $0.5 \mathrm{~mA} \mathrm{~cm}$, as well as a good capacitive response under a compressed state. This combination of compression strength and electrochemical properties makes this conducting silk hydrogel a potential material for unconventional energy storage applications.

\section{INTRODUCTION}

Naturally derived biopolymers such as silk, cellulose, and chitin have received increasing attention owing to their sustainability, biocompatibility, resource abundance, adjustable mechanical properties, and highly controllable postprocessing abilities. ${ }^{1}$ Among such, silk fibroin (SF) from spiders and silkworms possesses a hierarchical structural motif, giving rise to excellent mechanical performance, which can be used as multifunctional building scaffolds with widespread uses, including optical, environmental, biomedical, and cell guidance applications. ${ }^{2-4}$ On the other hand, electronically conducting polymers (ECPs), such as polypyrrole, polyaniline, and poly(3,4ethylene dioxythiophene), have been extensively investigated in efficient energy storage systems due to their adjustable electronic conductivity and electrochemical activity. ${ }^{5}$ As a result, a combination of these biopolymers and ECPs, in principle, has significant inherent advantages regarding biocompatibility, environment friendliness, renewability, low cost, mechanical robustness, electronic conductivity, electroactivity, and versatility. However, unlike their counterparts where the cellulose and chitin have been readily integrated with various ECPs for sustainable energy storage applica- tions, ${ }^{6-8}$ very few silk-based electrochemically active composites have been developed along this line. Therefore, an investigation of a combination of SF with ECPs toward electrochemically active silk composites is highly appealing for emerging sustainable energy applications.

For practical applications, both SF hydrogels ${ }^{9-12}$ and ECP hydrogels ${ }^{13-16}$ represent a class of soft materials with particular features providing a rational approach for developing smart materials with flexible and biocompatible interface and high permeability to ions. The fabrication of SF/ECP hydrogels can theoretically combine all of the properties of SF, ECPs, and hydrogels; hence, SF/ECP hydrogels can be attractive functional materials for applications in actuators, tissue engineering, bone regeneration, and energy storage. ${ }^{4,9-11,17}$

Received: January 22, 2020

Revised: April 23, 2020

Accepted: April 24, 2020

Published: April 24, 2020

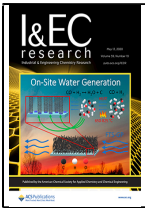


Table 1. PEDOT:PSS-SF Hydrogels Prepared Using Different Volumes of PEDOT:PSS Solution and the Corresponding Modulus (Mean of Three Measurements; Errors Bars Are S.D.)

\begin{tabular}{|c|c|c|c|c|c|c|}
\hline \multirow[b]{2}{*}{ group ID } & \multirow[b]{2}{*}{ SF solution $(\mathrm{mL})$} & \multirow[b]{2}{*}{ PEDOT:PSS solution (mL) } & \multicolumn{2}{|c|}{ storage modulus $\left(G^{\prime}\right)$} & \multicolumn{2}{|c|}{ loss modulus $\left(G^{\prime \prime}\right)$} \\
\hline & & & $10 \mathrm{rad} \mathrm{s}^{-1}$ & $0.1 \mathrm{rad} \mathrm{s}^{-1}$ & $10 \mathrm{rad} \mathrm{s}^{-1}$ & $0.1 \mathrm{rad} \mathrm{s}^{-1}$ \\
\hline G1 & 7.5 & 30 & $12950 \pm 560$ & $7213 \pm 390$ & $3606 \pm 478$ & $1741 \pm 111$ \\
\hline G2 & 7.5 & 22.5 & $16100 \pm 720$ & $8055 \pm 795$ & $3775 \pm 678$ & $1959 \pm 48$ \\
\hline G3 & 7.5 & 15 & $17120 \pm 690$ & $8662 \pm 558$ & $4049 \pm 474$ & $2230 \pm 70$ \\
\hline G4 & 7.5 & 7.5 & $18220 \pm 860$ & $10810 \pm 540$ & $5659 \pm 489$ & $2853 \pm 209$ \\
\hline
\end{tabular}

Although several hybrid SF/ECP composites have been explored, most reported SF/ECP hybrids are in the form of membranes where ECPs are deposited onto SF substrates, ${ }^{4,18-21}$ and only exceptional studies have been conducted where ECPs are introduced into SF hydrogels for cell manipulation and stimulation, ${ }^{10}$ sensing platform, ${ }^{2-24}$ actuator materials, ${ }^{25,26}$ and electrode materials. ${ }^{27}$

Herein, we report a novel approach to fabricate electrochemically active hydrogels based on an interpenetrating network of SF and ECP hydrogels with remarkable mechanical performance and good charge storage capacity. In this strategy, $\mathrm{SF}$ is initially dissolved in a $\mathrm{CaCl}_{2}-$ formic acid solvent to produce a $\mathrm{Ca}^{2+}-\mathrm{SF}$ solution; ${ }^{28,29}$ then, a commercial PEDOT:PSS suspension is introduced to the solution. A PEDOT:PSS gel first forms due to the cross-linking of PSS chains by $\mathrm{Ca}^{2+}$ ions, ${ }^{30}$ followed by further gelation due to the hydrophobic properties of SF $\beta$-sheets that form in formic acid aqueous solution after washing the gel to remove excess $\mathrm{Ca}^{2+}$ ions, ${ }^{31}$ producing an electrochemically active, conducting SF hydrogel (denoted as PEDOT:PSS-SF hydrogel). Compared to previously fabricated robust PEDOT:PSS hydrogels, ${ }^{32}$ the PEDOT:PSS-SF hydrogel maintains mechanical integrity even when compressed with $50 \%$ strain. A symmetric charge storage device based on conductive silk hydrogel electrodes exhibited a remarkable areal capacitance of $1.1 \mathrm{~F} \mathrm{~cm}^{-2}$ at 0.5 $\mathrm{mA} \mathrm{cm} \mathrm{cm}^{-2}$, as well as good capacitive response under a compressed state.

\section{EXPERIMENTAL SECTION}

2.1. Preparation of $\mathrm{SF} / \mathrm{CaCl}_{2} / \mathrm{FA}$ Solution. Bombyx mori was degummed based on a published protocol. ${ }^{2}$ Briefly, B. mori was boiled in a $0.02 \mathrm{M} \mathrm{Na}_{2} \mathrm{CO}_{3}$ solution for $30 \mathrm{~min}$. The obtained silk fibers were washed with deionized water to remove sericin and then dried at room temperature. Then, $3 \mathrm{~g}$ of dried fibers were dissolved in $20 \mathrm{~g}$ of formic acid containing $1 \mathrm{~g}$ of $\mathrm{CaCl}_{2}$ for a few minutes and then sonicated for $2 \mathrm{~min}$ to remove bubbles. ${ }^{33}$

2.2. Synthesis of PEDOT:PSS-SF Hydrogels. Four groups were synthesized by adding varying volumes of a PEDOT:PSS (PH 1000, Heraeus Clevios) solution dropwise into $7.5 \mathrm{~mL}$ of an $\mathrm{SF} / \mathrm{CaCl}_{2} / \mathrm{FA}$ solution (see Table 1 ). A gel immediately formed due to $\mathrm{Ca}^{2+}$ cross-linking of PSS and was allowed to set overnight. Finally, the gel was immersed in 30 $\mathrm{mL}$ of fresh deionized water for $30 \mathrm{~min}$ and then five times to remove the solvent totally, resulting in a robust hydrogel after $3 \mathrm{~h}$ due to an increased $\beta$-sheet content in SF. Although a small fraction of the compound was lost during water washing, the final hydrogels exhibited similar properties in each group.

2.3. Scanning Electron Microscopy (SEM). SEM was performed by an FEI Merlin equipped with an energydispersive spectrometer. Samples were lyophilized and coated with a thin $\mathrm{Au} / \mathrm{Pd}$ layer before analysis.
2.4. X-ray Photoelectron Spectroscopy (XPS). XPS was done using a PHI 5000 VersaProbe (ULVAC-PHI) equipped with $\mathrm{Al} \mathrm{K} \alpha \mathrm{X}$-ray source

2.5. Rheological Characterization. The rheological characterizations were carried out using an AR2000 Advanced Rheometer with an aluminum parallel-plate geometry of $8 \mathrm{~mm}$ diameter at room temperature. After reaching the linear viscoelastic regime by strain sweeps at $1 \mathrm{~Hz}$ frequency, the experiments were conducted at a fixed force $(0.03 \mathrm{~N})$ and a fixed strain (1\%) with frequency between 0.1 and $10 \mathrm{~Hz}$.

2.6. Fourier Transform Infrared (FTIR) Spectroscopy. The FTIR spectra of lyophilized hydrogels were recorded using a Bruker IFS 66v/S and collected from 4000 to $400 \mathrm{~cm}^{-1}$ with a resolution of $4 \mathrm{~cm}^{-1}$. Background removal was performed using a blank scan.

2.7. Raman Spectroscopy. The Raman spectroscopy studies of lyophilized hydrogels were performed by a Renishaw Ramascope. Before analysis, a silicon wafer at a $521 \mathrm{~cm}^{-1}$ band was used to calibrate the Raman instrument.

2.8. Mechanical Tests. The compression tests were performed by a universal equipment (AGS-X, Shimadzu) at $25 \pm 1{ }^{\circ} \mathrm{C}$. The PEDOT:PSS-SF hydrogels were cut into cubic specimens $(5 \mathrm{~mm} \times 5 \mathrm{~mm} \times 10 \mathrm{~mm})$. The test of each sample was repeated five times. The loading velocity was $1 \mathrm{~mm}$ $\min ^{-1}$.

2.9. Conductivity. The conductivity of each PEDOT:PSS-SF hydrogel was measured by a four-point probe.

2.10. CV Measurements. The CV measurements were performed in a standard three-electrode electrochemical cell, in which a piece of PEDOT:PSS-SF hydrogel, a Pt wire, and an $\mathrm{Ag} / \mathrm{AgCl}$ electrode were used as the working electrode, counter electrode, and reference electrode, respectively. A 1 $\mathrm{M} \mathrm{Na} \mathrm{SO}_{4}$ solution was purged with $\mathrm{N}_{2}$ for $5 \mathrm{~min}$ and employed as the electrolyte. The charge capacity was calculated based on the weight of the electrode. AC impedance was measured on a $\mathrm{CH}$ Instruments $660 \mathrm{D}$ potentiostat $(\mathrm{CH}$ Instruments, Inc.) at a cell potential of $0 \mathrm{~V}$, using an ac amplitude of $10 \mathrm{mV}$ in the frequency range of $100 \mathrm{kHz}$ to 0.01 $\mathrm{Hz}$.

2.11. Fabrication of Supercapacitor Devices. The supercapacitor devices were fabricated as follows: Two circular pieces of the hydrogel electrode were laminated onto two graphite foil current collectors and then separated by an ordinary filter paper separator. A $1 \mathrm{M} \mathrm{Na}_{2} \mathrm{SO}_{4}$ electrolyte was added before measurement. The supercapacitors were assembled and sealed in a coffee bag. The cells were charged/discharged at various current densities between 0.5 and $10 \mathrm{~mA} \mathrm{~cm}{ }^{-2}$, while cycled at a constant current density of $2 \mathrm{~mA} \mathrm{~cm}{ }^{-2}$ between 0 and $0.8 \mathrm{~V}$.

2.12. Cell Capacitance. The cell capacitance was derived from the galvanostatic discharge data according to $C=I \Delta t$ / $\Delta V$, where $C, I$, and $\Delta V$ refers to the cell capacitance, discharge current, and potential shift within the discharge time $\Delta t(\mathrm{~s})$, 


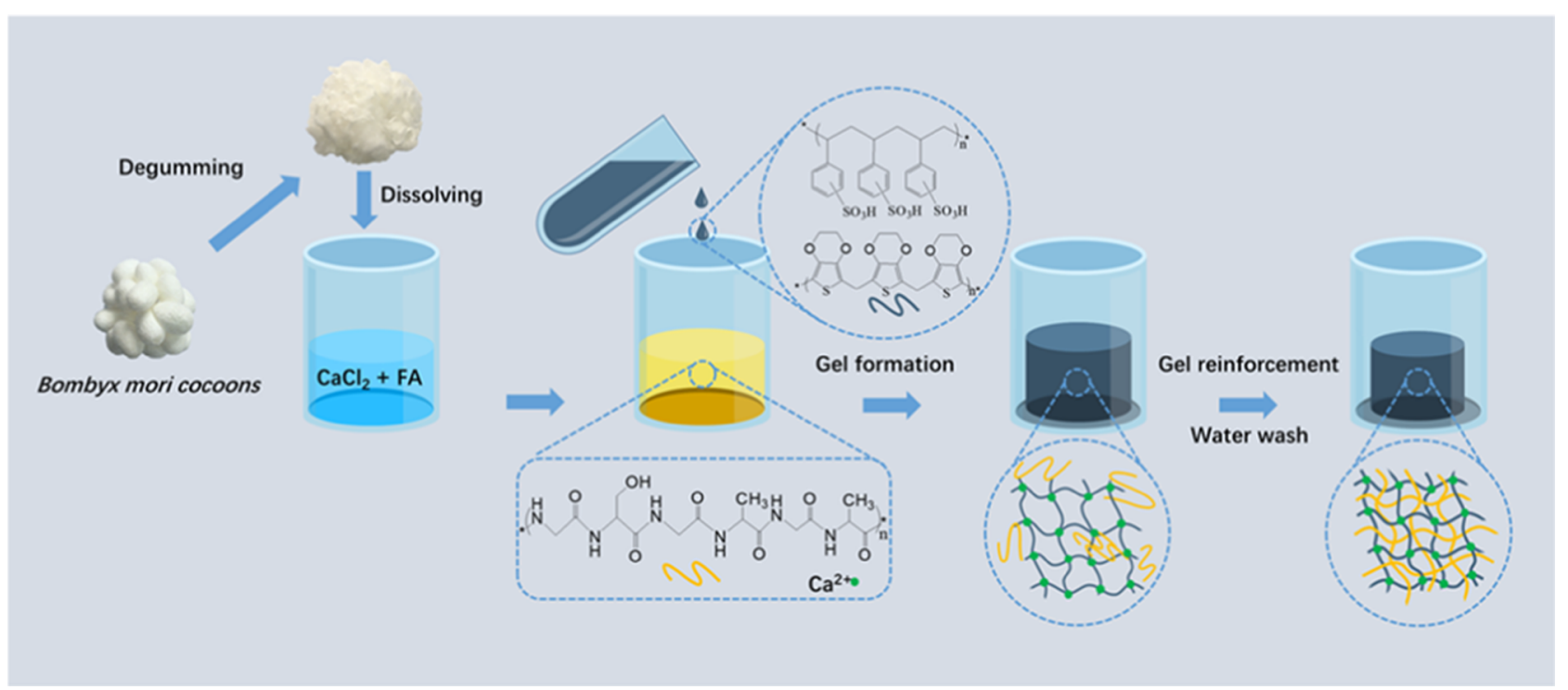

Figure 1. Schematic illustration of the synthesis process of PEDOT:PSS-SF hydrogels.

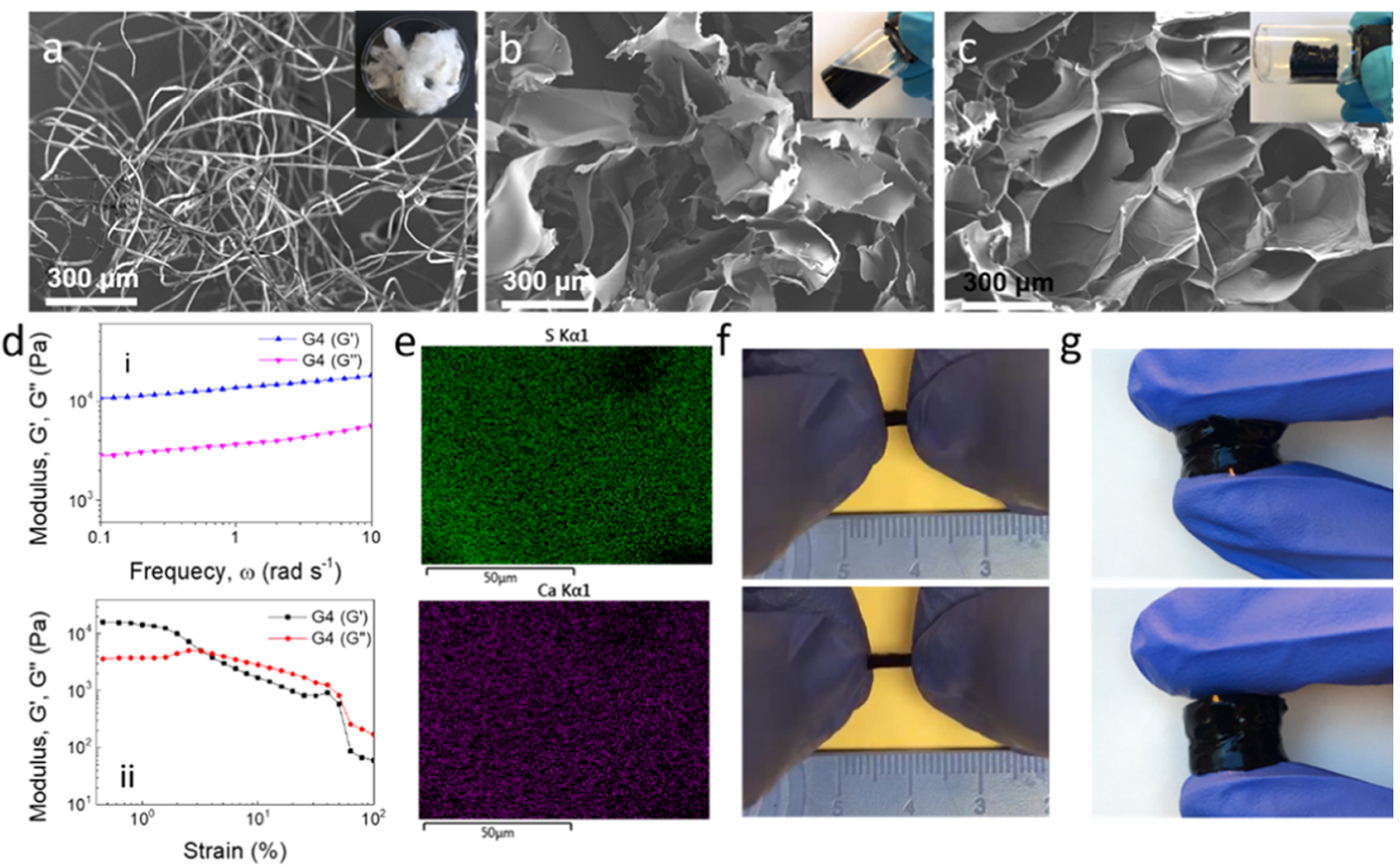

Figure 2. Morphology and structure of PEDOT:PSS-SF hydrogels. SEM images of (a) degummed silk fibers, (b) lyophilized PEDOT:PSS solution, and (c) lyophilized hydrogel G4. (d) Rheological test results of G4. (e) EDS of Ca and S for G4. G4 when mechanically (f) stretched and (g) compressed.

respectively. The area-normalized cell capacitance was estimated as $C_{\mathrm{s}}=\mathrm{C} / \mathrm{S}$, where $S$ denotes the geometric area of the electrodes. While we noted that low active mass content in a dried hydrogel (about 6 wt \% PEDOT) and large thickness of hydrogels (typically $350 \mu \mathrm{m}$ ) are not practical in real energy storage devices, our studies are fundamental in nature and our conclusions are not influenced by the capacitance in the conductive SF hydrogels.

\section{RESULTS AND DISCUSSION}

3.1. PEDOT:PSS-SF Hydrogel Preparation. The preparation principle of the conducting PEDOT:PSS-SF hydrogel is illustrated in Figure 1. First, pure SF is obtained after degumming the B. mori cocoons to acts as a starting material. Then, SF is added into a $\mathrm{CaCl}_{2}$ and formic acid binary solvent, giving rise to a yellowish SF solution (Figure $\mathrm{S} 1$ ), in which $\mathrm{Ca}^{2+}$ ions inhibit the $\beta$-sheet formation of SF chains. ${ }^{33} \mathrm{~A}$ PEDOT:PSS solution is then introduced into the SF solution, which induces gelation of a PEDOT:PSS hydrogel immediately in the SF solution due to the cross-linking of $\mathrm{Ca}^{2+}$ ions with the negatively charged PSS ionomers via electrostatic interaction. ${ }^{34}$ A similar phenomenon is also found when a $\mathrm{CaCl}_{2}$ aqueous solution is mixed with a PEDOT:PSS solution (Figure S2). Finally, a water wash removes free $\mathrm{Ca}^{2+}$ ions in the SF solution, allowing SF $\beta$-sheet formation and further gelation of the hydrogel due to hydrophobic aggregates of incorporated SF to 
get PEDOT:PSS-SF hydrogels. Further synthesis details and composition of the PEDOT:PSS-SF hydrogels can be found in the Experimental Section and Table S1. It is found that the viscosity of the starting mixture increased dramatically along with a higher SF content, which gives rise to an uneven distribution of PEDOT:PSS during hydrogel formation. The hydrogel, in which the ratio of SF to PEDOT:PSS is 7.5:5, is nonuniform and shows unstable mechanical properties. Therefore, hydrogels with a higher SF content than sample G4 are not used.

3.2. Formation and Characterization of PEDOT:PSSSF Hydrogels. The preparation procedure involves the integration of two precursors with different morphologies, as shown in Figure 2a,b. The pristine SF has a fibrous structure with fiber widths on the micron scale (Figure 2a). While the lyophilized PEDOT:PSS (Figure 2b) and PEDOT:PSS-SF hydrogel (G4, Figure 2c) samples both show interconnected leaflike structures, the PEDOT:PSS sample appears to have a more irregular pore structure with thinner walls than the PEDOT:PSS-SF hydrogel sample. Note that the sample G4 in Figure $2 c$ had the highest SF content in the four prepared hydrogel groups (cf. Table 1). Other PEDOT:PSS-SF hydrogels exhibited a similar structure to G4 (Figure S3). S and $\mathrm{N}$ elements from PEDOT:PSS and SF, respectively, are detected by XPS (Figure S4), and the elemental mapping of G4 was conducted using EDS (Figure 2e), which shows a uniform distribution of $\mathrm{S}$ and $\mathrm{Ca}$ elements, indicating the cross-linking effect. ${ }^{32}$ These results suggest an interpenetrated structure of the PEDOT:PSS-SF hydrogel, and the structure can improve the mechanical strength of hydrogels. ${ }^{35}$ As shown in Figures $2 \mathrm{~d}$ and S5, the rheological properties of PEDOT:PSS-SF hydrogels are assessed in the range of $0.1-$ $10 \mathrm{rad} \mathrm{s}^{-1}$ under isothermal conditions. The storage modulus $G^{\prime}$ and the loss modulus $G^{\prime \prime}$ provide information on the energy change. The storage modulus $G^{\prime}$ is higher than the corresponding loss modulus $G^{\prime \prime}$, suggesting that the PEDOT:PSS-SF hydrogel possesses characteristics of a viscoelastic solid. Interestingly, the modulus of the hydrogels increases with SF content in all groups, where the corresponding $G^{\prime}$ increases from $7213 \pm 390$ to $10810 \pm$ $540 \mathrm{~Pa}$, and $G^{\prime \prime}$ from $1741 \pm 111$ to $2853 \pm 209 \mathrm{~Pa}$ at a frequency of $0.1 \mathrm{rad} \mathrm{s} \mathrm{s}^{-1}$ for hydrogels of G1 and G4, respectively (Table 1 ), indicating that $\mathrm{SF}$ in the interpenetrated network is beneficial to the mechanical strength of the hydrogels. The enhanced mechanical strength of the hydrogels along with increased SF content is due to the secondary structural changes of SF from more random coils to more $\beta$-sheet forms. ${ }^{36}$ All of the PEDOT:PSS-SF hydrogels showed a much higher mechanical strength than PEDOT:PSS hydrogels cross-linked by metal ions. ${ }^{32}$ The PEDOT:PSS-SF hydrogels were liquefied at a shear strain above $\approx 10 \%$, which is proved by the lower storage modulus $G^{\prime}$ than loss modulus $G^{\prime \prime}$ (Figures 2d,ii and S6). The shear-thinning property is beneficial for the injectability of the materials and a full recovery of the initial state post injection. This indicates that the PEDOT:PSS-SF hydrogels have a potential as injectable electronic devices. ${ }^{37}$ Interestingly, G4 (i.e., the highest SF content in all groups) was found to recover completely after deformation, even when stretched above 100\% strain (Figure $2 \mathrm{f}$ ) or compressed above $50 \%$ strain (Figure $2 \mathrm{~g}$ ). The mechanical strengths of PEDOT:PSS-SF hydrogels are greater than those of many other types of PEDOT:PSS hydrogels, ${ }^{32}$ and the storage moduli are tens to hundreds of times higher than those of conventional biopolymer hydrogels, ${ }^{15}$ suggesting that the PEDOT:PSS-SF hydrogels could be used in applications for pressure- and flexion-sensitive electronic devices. ${ }^{38}$

3.3. Composition of PEDOT:PSS-SF Hydrogels. To further determine the composition of the PEDOT:PSS-SF hydrogels, FTIR and Raman spectroscopy experiments were performed on PEDOT:PSS, SF, and PEDOT:PSS-SF hydrogels. The characteristic silk peaks appeared in the FTIR spectra (Figure 3a,b) for the lyophilized PEDOT:PSS-SF hydrogels,
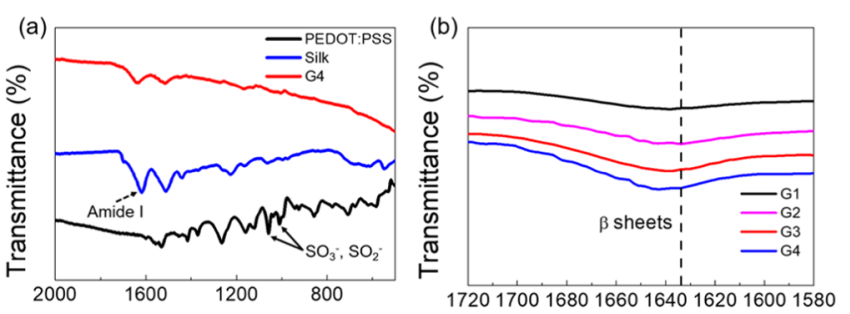

Wavenumber $\left(\mathrm{cm}^{-1}\right)$
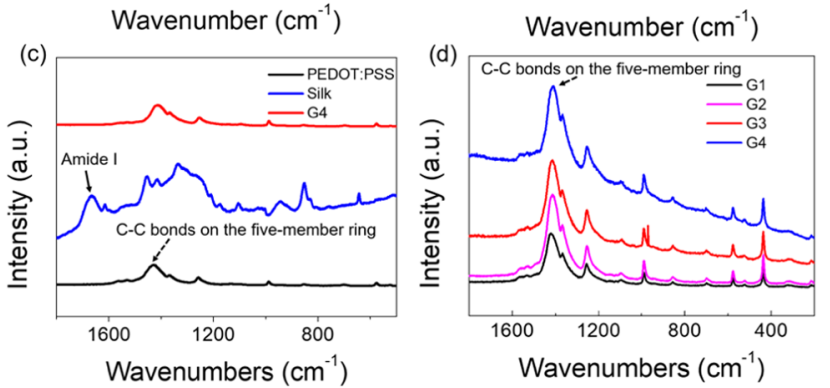

Figure 3. (a) FTIR spectra and (c) Raman spectra of silk, lyophilized PEDOT:PSS solution, and lyophilized PEDOT:PSS-SF hydrogels (G4). (b) FTIR and (d) Raman spectra of PEDOT:PSS-SF hydrogels G1, G2, G3, and G4.

e.g., amide I bonds (dashed arrow in Figure 3a). It demonstrates that PEDOT:PSS-SF hydrogels include silk fibroin. In addition, two well-defined characteristic peaks were observed at 1058 and $1010 \mathrm{~cm}^{-1}$ (solid arrows in Figure 3a), which can be ascribed to the symmetric stretching of $\mathrm{SO}_{3}{ }^{-}$and $\mathrm{SO}_{2}{ }^{-}$. However, these two characteristic PEDOT:PSS peaks did not appear in the FTIR spectrum for G4, which is most likely due to the overlap of energy absorbed by SF at these frequencies. While $\mathrm{Ca}^{2+}$ ions can inhibit the structural transformation of SF from random coils to $\beta$-sheets in the $\mathrm{SF}-\mathrm{CaCl}_{2}-\mathrm{FA}$ solution, ${ }^{33}$ the removal of $\mathrm{Ca}^{2+}$ in the final washing step during the synthesis process results in more $\beta$ sheet formation and subsequent gelation of the PEDOT:PSSSF hydrogel due to hydrophobic effects. Notably, the peak area corresponding to $\beta$-sheets (dashed lines) increases with SF content (Figure 3b), which agrees with the increased mechanical strength in the rheological characterization.

The hydrogels were further examined by Raman spectroscopy (Figure $3 \mathrm{c}, \mathrm{d}$ ). The characteristic peak of SF, corresponding to the amide I band (solid arrow in Figure 3c), did not appear in the spectra of lyophilized PEDOT:PSS-SF hydrogels due to the much lower absorbance intensity of SF compared to PEDOT:PSS. A slight difference between PEDOT:PSS and G4 is observed for the strong band located between 1400 and $1500 \mathrm{~cm}^{-1}$, corresponding to the stretching vibration of $\mathrm{C}-\mathrm{C}$ bonds on the five-membered ring of PEDOT (dashed arrow in Figure 3c). ${ }^{39}$ Compared to the spectrum of PEDOT:PSS, the band between 1400 and $1500 \mathrm{~cm}^{-1}$ for G4 
becomes slightly narrower and shifted to lower wavenumbers, which suggests that the PEDOT polymer chains in PEDOT:PSS-SF hydrogels possess a more linear or expanded-coil conformation compared to those in PEDOT:PSS hydrogels. It can result in improved $\pi-\pi$ interchain stacking, leading to an elongation of the effective conjugation length and a longer mean free path, which are important for electrochemical applications. ${ }^{39}$ No obvious difference can be found between the spectra of the PEDOT:PSS-SF hydrogels in the Raman band between 1400 and $1500 \mathrm{~cm}^{-1}$ (Figure 3d), which indicates a similar PEDOT conformation in PEDOT:PSS-SF hydrogels in all groups.

3.4. Compressive Properties of PEDOT:PSS-SF Hydrogels. As described above, the PEDOT:PSS-SF hydrogel has the potential to be used as a compressible electrode material. To investigate the compressibility of the materials, model electrodes composed of PEDOT:PSS-SF hydrogels were subjected to repetitive compression experiments. Representative stress-strain curves for a continuous, increasing application of compressive stress leading to a strain of $30 \%$ and then reducing the stress back to $0 \mathrm{kPa}$ are plotted in Figure 4a for G1 to G4. When the SF content increased from $78.9 \mathrm{wt}$ $\%$ (G1) to 93.8 wt \% (G4), the required compressive stress to produce a $30 \%$ strain increased from $6.6 \pm 0.7$ to $13.6 \pm 1.1$ $\mathrm{kPa}$. All samples exhibited a pronounced hysteresis with a residual strain between 13 and $20 \%$ at completion of the cycle. However, all samples showed $100 \%$ length recovery in $30 \mathrm{~min}$ after completion of the compression test.
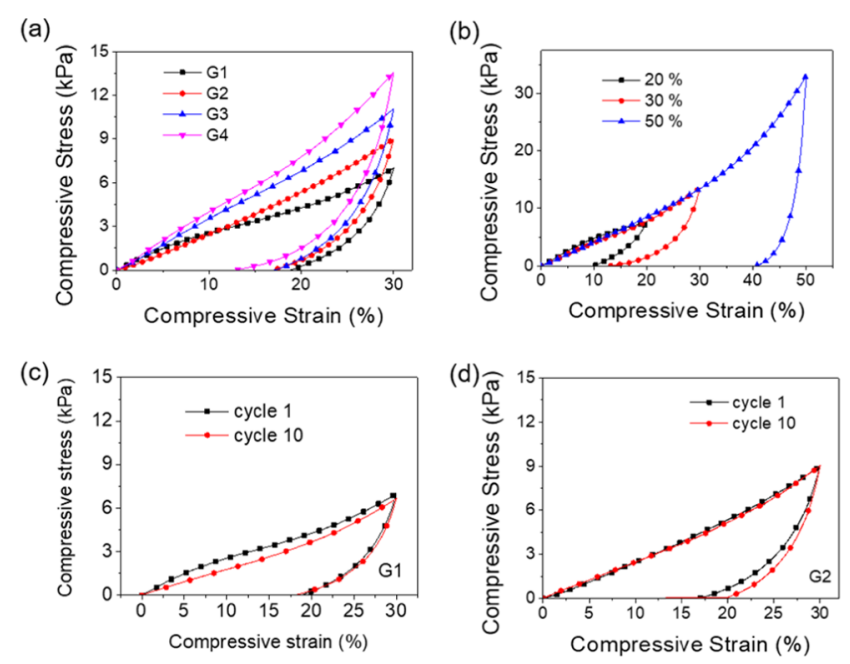

(e)
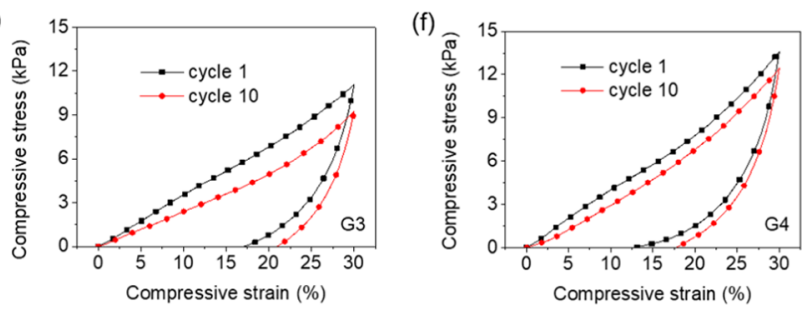

Figure 4. Mechanical properties of PEDOT:PSS-SF hydrogels. (a) Self-recovery curves of PEDOT:PSS-SF hydrogels under compressive loading (mean of five measurements). (b) Typical stress-strain curves of G4 with different maximum strains under compressive loading-unloading tests. Typical stress-strain curves of PEDOT:PSS-SF hydrogels under cyclic compressive loading and unloading tests for the first and tenth cycles for G1 (c), G2 (d), G3 (e), and G4 (f).
The robustness of the hydrogels is further exemplified by testing the G4 with a $93.8 \%$ SF content under three consecutive loading-unloading cycles at maximum strains of 20,30 , and $50 \%$ and applying $30 \mathrm{~min}$ rest between cycles (Figure 4b). A stress of $32.9 \pm 1.9 \mathrm{kPa}$ was required to produce $50 \%$ strain, and the hysteresis loop increased compared to the cycles of lower strain. Even after the cycle with $50 \%$ strain, the hydrogel recovered its original length in $30 \mathrm{~min}$ after completion of the test, indicating that the structure of the hydrogel is very stable. Repeated loadingunloading tests were performed at a strain of $30 \%$ to further assess the self-recovery behavior of the PEDOT:PSS-SF hydrogels under compression conditions (see Figure $4 \mathrm{c}-\mathrm{f}$ for G1, G2, G3, and G4, respectively). It was observed that the PEDOT:PSS-SF hydrogels produced similar strain-stress curves through 10 cycles when 30 min resting time was applied before each compression loading. It can be ascribed to the interpenetrated network between SF and PEDOT:PSS, the mechanical reinforcement provided by the cross-linking with $\mathrm{Ca}^{2+}$ ions, and the inherent mechanical strength of both SF and PEDOT:PSS. Conventional hydrogels and other "soft" biocompatible materials can be divided into two broad classes according to Miserez et al.: ${ }^{40}$ either they are stiff with low failure modulus, such as native collagen, ${ }^{41}$ or they are elastic with limited modulus, as in the case of alginate. ${ }^{42}$ In the current study, the PEDOT:PSS-SF hydrogels show a high modulus and elastic properties simultaneously, and a $100 \%$ recovery can be realized after at least $50 \%$ strain due to compressive stress.

3.5. Electrochemical Properties and Conductivity of Compressible PEDOT:PSS-SF Hydrogels. Due to the combination of good conductivity, measured at $1 \mathrm{mS} \mathrm{cm}$, with a four-point probe, and the above-described mechanical properties, our PEDOT:PSS-SF hydrogels have shown potential for application in energy storage, sensors, and actuators. Herein, sample G4 was selected as a supercapacitor electrode to evaluate the charge storage property owing to its good compressive ability. The charge storage capacity of the conducting PEDOT:PSS-SF hydrogel was studied with a symmetrical supercapacitor configuration with the $1 \mathrm{M}$ $\mathrm{Na}_{2} \mathrm{SO}_{4}$ electrolyte. The cyclic voltammograms of the silkbased supercapacitor showed rectangular shapes under various scan rates, and no faradic oxidation and reduction peaks were observed as seen in Figure 5a. This pseudocapacitive behavior agrees well with previous findings for similar PEDOT-based electrodes, ${ }^{43}$ as well as the nearly linear galvanostatic charge and discharge curves (Figure 5b). The approximate linear voltage profiles and the symmetrical charge/discharge curves indicate good capacitive response for the PEDOT:PSS-SF hydrogel electrode. The specific capacitance of the hydrogel electrode is presented in Figure 5c. It can be seen that the specific capacitance decreased with increased current densities; nevertheless, the specific capacitance per area for the $200 \mu \mathrm{m}$ thick PEDOT:PSS-SF electrodes exhibited an areal capacitance of $1.1 \mathrm{~F} \mathrm{~cm}^{-2}$ at $0.5 \mathrm{~mA} \mathrm{~cm}{ }^{-2}\left(77 \mathrm{~F} \mathrm{~g}^{-1}\right)$ and $0.3 \mathrm{~F} \mathrm{~cm}^{-2}$ at $10 \mathrm{~mA} \mathrm{~cm}$. The latter value is comparable to those of previously reported ECP hydrogel-based electrodes (e.g., PEDOT hydrogel, $0.115 \mathrm{~F} \mathrm{~cm}^{-2}$ at $0.92 \mathrm{~mA} \mathrm{~cm}^{-2}$, 44 and PANI/PVA hydrogel, $0.3 \mathrm{~F} \mathrm{~cm}^{-2}$ at $0.25 \mathrm{~A} \mathrm{~g}^{-145}$ ).

The PEDOT:PSS-SF hydrogel electrode was also studied using AC impedance measurements, as shown in the Nyquist plot in Figure S7. The very small semicircle (radius $\sim 0.2 \Omega$ ) followed by a straight line (with a slightly higher than $45^{\circ}$ 
(a)

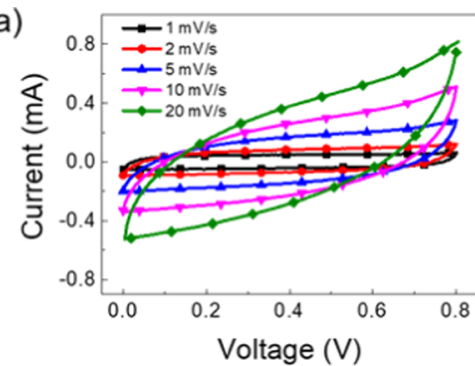

(c)

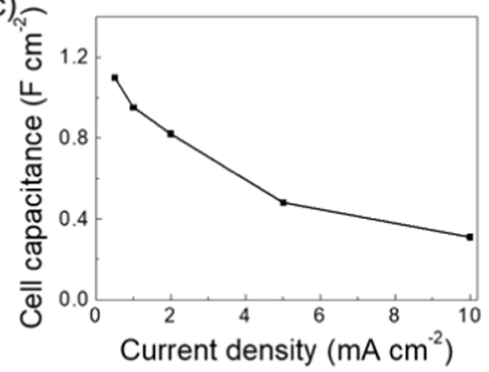

(b)

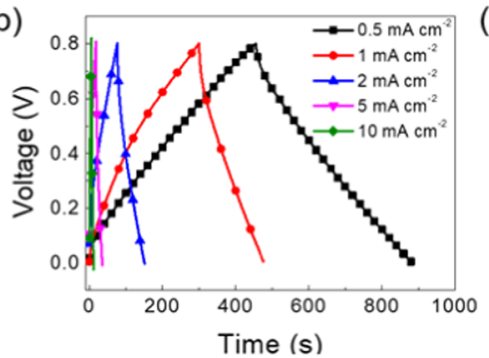

(e)

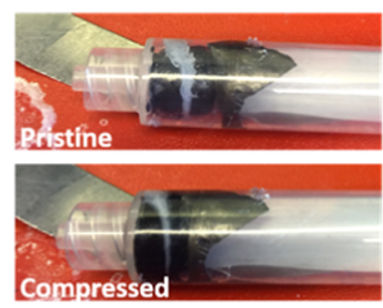

(d)

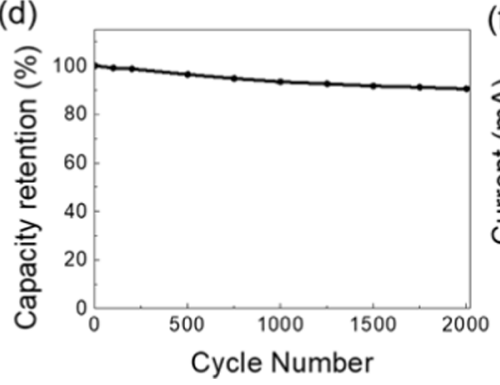

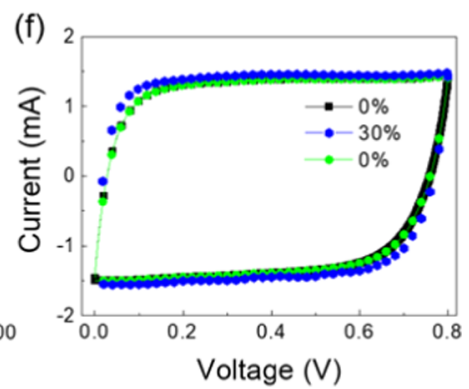

Figure 5. (a) Voltammograms recorded at various scan rates for the PEDOT:PSS-SF hydrogel electrode. (b) Galvanostatic charge/discharge profiles for the PEDOT paper electrode recorded at various current densities, (c) rate-dependent specific cell capacitance, and (d) long-term cycle stability for the conductive SF hydrogel electrode-based devices. (e) Optical photograph of the two-electrode setup and (f) the corresponding cyclic voltammograms under different compression states.

slope) in the Nyquist plot as well as a close-to-ideal capacitive behavior at the lowest frequencies are in good agreement with other common observations. ${ }^{43,46}$ The results of galvanostatic cycling experiments for the PEDOT:PSS-SF hydrogel electrode over 2000 cycles at a current density of $2 \mathrm{~mA}$ $\mathrm{cm}^{-2}$ are shown in Figure 5d. It demonstrated good cycling stability of the PEDOT:PSS-SF hydrogel as a capacitance retention of $90.5 \%$ was obtained upon 2000 cycles.

The high compressibility and good structural stability of the conducting PEDOT:PSS-SF hydrogel suggest the potential application as an electrode in compressible supercapacitors. As a proof of concept, we assembled two PEDOT:PSS-SF hydrogel electrodes, symmetrically paired with a filter paper separator and $1 \mathrm{M} \mathrm{Na}_{2} \mathrm{SO}_{4}$ electrolyte, into a compressible supercapacitor using a homemade instrument that can control the compressive strain of the hydrogel electrode (Figure 5e). The capacitive performance of the hydrogel electrode under compression conditions was evaluated by $\mathrm{CV}$ experiments recorded at a scan rate of $10 \mathrm{mV} \mathrm{s}^{-1}$. As can be seen in Figure $5 \mathrm{f}$, negligible changes were observed in the voltammograms recorded at two different supercapacitor compression states, i.e., 0 and $30 \%$, which indicates that the PEDOT:PSS-SF hydrogel electrodes hold great promise for compressible device applications.

\section{CONCLUSIONS}

In summary, we have developed a facile and novel approach to construct conducting silk fibroin hydrogels based on an interpenetrating network of PEDOT:PSS and silk fibroin. A combination of cross-linking PSS by $\mathrm{Ca}^{2+}$ ions and reinforcement by silk fibroin networks yields a robust and compressible composite, while PEDOT gives rise to conductivity and electrochemical activity. When employed in symmetric supercapacitor devices, the conductive silk hydrogel electrodes showed an areal capacitance of $1.1 \mathrm{~F} \mathrm{~cm}^{-2}$ at $0.5 \mathrm{~mA} \mathrm{~cm}$. Moreover, the high compressibility and good structural stability of the conducting silk hydrogels suggest their potential application as electrodes in compressible energy storage devices.

\section{ASSOCIATED CONTENT}

\section{Supporting Information}

The Supporting Information is available free of charge at https://pubs.acs.org/doi/10.1021/acs.iecr.0c00407.

Photo images of SF solution; PEDOT:PSS hydrogels; additional SEM images of PEDOT:PSS-SF hydrogels; XPS spectra of SF, PEDOT:PSS, and PEDOT:PSS-SF; additional rheological data of PEDOT:PSS-SF hydrogels; and additional electrochemical characterization of PEDOT:PSS-SF hydrogels (PDF)

\section{AUTHOR INFORMATION}

\section{Corresponding Author}

Zhaohui Wang - College of Materials Science and Engineering, Hunan University, Changsha 410082, China; Department of Chemistry-Ångström Laboratory, Uppsala University, Uppsala SE-75121, Sweden; 이이.org/0000-0001-6118-0226;

Email: zhaohui.wang@kemi.uu.se

\section{Authors}

Jiaojiao Yang - State Key Laboratory of Oral Diseases, West China Hospital of Stomatology, Sichuan University, Chengdu 610041, China; Department of Engineering Science, Uppsala University, Uppsala SE-75121, Sweden; (1) orcid.org/00000002-8611-4089

Jun Luo - College of Polymer Science and Engineering, Sichuan University, Chengdu 610065, China; Department of Engineering Science, Uppsala University, Uppsala SE-75121, Sweden; (1) orcid.org/0000-0001-6830-1633

Haidong Liu - Department of Chemistry-Ångström Laboratory, Uppsala University, Uppsala SE-75121, Sweden; 다. orcid.org/ 0000-0001-8519-3240 
Liyang Shi - State Key Laboratory of Chemo/Biosensing and Chemometrics, College of Biology, Hunan University, Changsha 410082, China; 이이이.0rg/0000-0002-2422-7280

Ken Welch - Department of Engineering Science, Uppsala University, Uppsala SE-75121, Sweden; ㅇoㅇid.org/00000003-4543-1130

Maria Strømme - Department of Engineering Science, Uppsala University, Uppsala SE-75121, Sweden; o orcid.org/00000002-5496-9664

Complete contact information is available at:

https://pubs.acs.org/10.1021/acs.iecr.0c00407

\section{Funding}

This work was supported by the National Natural Science Foundation of China (51903169), China Postdoctoral Science Foundation (2019M663529), Special Funding of State Key Laboratory of Oral Diseases (SKLOD202019), Postdoctoral Cross Funding of Sichuan University (0040304153059), and Research Funding for talents developing, West China Hospital of Stomatology, Sichuan University (RCDWJS2020-17).

\section{Notes}

The authors declare no competing financial interest.

\section{ACKNOWLEDGMENTS}

The authors thank Chenyu Wen and Zhen Qiu for four-point probe and Raman spectroscopy measurements, respectively.

\section{REFERENCES}

(1) Landry, M. J.; Rollet, F.-G.; Kennedy, T. E.; Barrett, C. J. Layers and Multilayers of Self-Assembled Polymers: Tunable Engineered Extracellular Matrix Coatings for Neural Cell Growth. Langmuir 2018, 34, 8709-8730.

(2) Rockwood, D. N.; Preda, R. C.; Yucel, T.; Wang, X.; Lovett, M. L.; Kaplan, D. L. Materials Fabrication from Bombyx mori Silk Fibroin. Nat. Protoc. 2011, 6, 1612-1631.

(3) Das, A. M.; Chowdhury, P. K.; Saikia, C. N.; Rao, P. G. Some Physical Properties and Structure Determination of Vinyl MonomerGrafted Antheraea Assama Silk Fiber. Ind. Eng. Chem. Res. 2009, 48, 9338-9345.

(4) Ambekar, R. S.; Kandasubramanian, B. Progress in the Advancement of Porous Biopolymer Scaffold: Tissue Engineering Application. Ind. Eng. Chem. Res. 2019, 58, 6163-6194.

(5) Nyholm, L.; Nyström, G.; Mihranyan, A.; Strømme, M. Toward Flexible Polymer and Paper-Based Energy Storage Devices. Adv. Mater. 2011, 23, 3751-3769.

(6) Jin, J.; Lee, D.; Im, H. G.; Han, Y. C.; Jeong, E. G.; Rolandi, M.; Choi, K. C.; Bae, B. S. Chitin Nanofiber Transparent Paper for Flexible Green Electronics. Adv. Mater. 2016, 28, 5169-5175.

(7) Ma, Q.; Pu, K. B.; Cai, W. F.; Wang, Y. H.; Chen, Q. Y.; Li, F. J. Characteristics of Poly(3, 4-ethylenedioxythiophene) Modified Stainless Steel as Anode in Air-Cathode Microbial Fuel Cells. Ind. Eng. Chem. Res. 2018, 57, 6633-6638.

(8) Wang, Z. H.; Tammela, P.; Stromme, M.; Nyholm, L. CelluloseBased Supercapacitors: Material and Performance Considerations. Adv. Energy Mater. 2017, 7, No. 1700130.

(9) Zhu, Z.; Ling, S.; Yeo, J.; Zhao, S.; Tozzi, L.; Buehler, M. J.; Omenetto, F.; Li, C.; Kaplan, D. L. High-Strength, Durable All-Silk Fibroin Hydrogels with Versatile Processability toward Multifunctional Applications. Adv. Funct. Mater. 2018, 28, No. 1704757.

(10) Teshima, T.; Nakashima, H.; Kasai, N.; Sasaki, S.; Tanaka, A.; Tsukada, S.; Sumitomo, K. Mobile Silk Fibroin Electrode for Manipulation and Electrical Stimulation of Adherent Cells. Adv. Funct. Mater. 2016, 26, 8185-8193.

(11) Shi, L.; Wang, F.; Zhu, W.; Xu, Z.; Fuchs, S.; Hilborn, J.; Zhu, L.; Ma, Q.; Wang, Y.; Weng, X.; Ossipov, D. A. Self-Healing Silk
Fibroin-Based Hydrogel for Bone Regeneration: Dynamic MetalLigand Self-Assembly Approach. Adv. Funct. Mater. 2017, 27, No. 1700591.

(12) Li, Z.; Zheng, Z.; Yang, Y.; Fang, G.; Yao, J.; Shao, Z.; Chen, X. Robust Protein Hydrogels from Silkworm Silk. ACS Sustainable Chem. Eng. 2016, 4, 1500-1506.

(13) Wang, Z.; Tammela, P.; Zhang, P.; Huo, J.; Ericson, F.; Strømme, M.; Nyholm, L. Freestanding Nanocellulose-Composite Fibre Reinforced 3D Polypyrrole Electrodes for Energy Storage Applications. Nanoscale 2014, 6, 13068-13075.

(14) Zhou, K.; He, Y.; Xu, Q.; Zhang, Q.; Zhou, A.; Lu, Z.; Yang, L.K.; Jiang, Y.; Ge, D.; Liu, X. Y.; Bai, H. A Hydrogel of Ultrathin Pure Polyaniline Nanofibers: Oxidant-Templating Preparation and Supercapacitor Application. ACS Nano 2018, 12, 5888-5894.

(15) Ding, H.; Zhong, M.; Kim, Y. J.; Pholpabu, P.; Balasubramanian, A.; Hui, C. M.; He, H.; Yang, H.; Matyjaszewski, K.; Bettinger, C. J. Biologically Derived Soft Conducting Hydrogels Using Heparin-Doped Polymer Networks. ACS Nano 2014, 8, 43484357.

(16) Wang, X.; Shu, J.-C.; He, X.-M.; Zhang, M.; Wang, X.-X.; Gao, C.; Yuan, J.; Cao, M.-S. Green Approach to Conductive PEDOT:PSS Decorating Magnetic-Graphene to Recover Conductivity for Highly Efficient Absorption. ACS Sustainable Chem. Eng. 2018, 6, 1401714025 .

(17) Jin, H.-J.; Park, J.; Valluzzi, R.; Cebe, P.; Kaplan, D. L. Biomaterial Films of Bombyx mori Silk Fibroin with Poly(ethylene oxide). Biomacromolecules 2004, 5, 711-717.

(18) Aznar-Cervantes, S.; Roca, M. I.; Martinez, J. G.; MeseguerOlmo, L.; Cenis, J. L.; Moraleda, J. M.; Otero, T. F. Fabrication of Conductive Electrospun Silk Fibroin Scaffolds by Coating with Polypyrrole for Biomedical Applications. Bioelectrochemistry 2012, 85, 36-43.

(19) Romero, I. S.; Schurr, M. L.; Lally, J. V.; Kotlik, M. Z.; Murphy, A. R. Enhancing the Interface in Silk-Polypyrrole Composites through Chemical Modification of Silk Fibroin. ACS Appl. Mater. Interfaces 2013, 5, 553-564.

(20) Pal, R. K.; Farghaly, A. A.; Collinson, M. M.; Kundu, S. C.; Yadavalli, V. K. Photolithographic Micropatterning of Conducting Polymers on Flexible Silk Matrices. Adv. Mater. 2016, 28, 1406-1412.

(21) Jia, X.; Wang, C.; Zhao, C.; Ge, Y.; Wallace, G. G. Toward Biodegradable Mg-Air Bioelectric Batteries Composed of Silk FibroinPolypyrrole Film. Adv. Funct. Mater. 2016, 26, 1454-1462.

(22) He, F.; You, X.; Gong, H.; Yang, Y.; Bai, T.; Wang, W.; Guo, W.; Liu, X.; Ye, M. Stretchable, Biocompatible, and Multifunctional Silk Fibroin-Based Hydrogels toward Wearable Strain/Pressure Sensors and Triboelectric Nanogenerators. ACS Appl. Mater. Interfaces 2020, 12, 6442-6450.

(23) Chen, F.; Lu, S.; Zhu, L.; Tang, Z.; Wang, Q.; Qin, G.; Yang, J.; Sun, G.; Zhang, Q.; Chen, Q. Conductive Regenerated Silk-FibroinBased Hydrogels with Integrated High Mechanical Performances. J. Mater. Chem. B 2019, 7, 1708-1715.

(24) Yang, N.; Qi, P.; Ren, J.; Yu, H.; Liu, S.; Li, J.; Chen, W.; Kaplan, D.; Ling, S. Polyvinyl Alcohol/Silk Fibroin/Borax Hydrogel Ionotronics: A Highly Stretchable, Self-Healable, and Biocompatible Sensing Platform. ACS Appl. Mater. Interfaces 2019, 11, 2363223638 .

(25) Srisawasdi, T.; Petcharoen, K.; Sirivat, A.; Jamieson, A. Electromechanical Response of Silk Fibroin Hydrogel and Conductive Polycarbazole/Silk Fibroin Hydrogel Composites as Actuator Material. Mater. Sci. Eng., C 2015, 56, 1-8.

(26) Yao, M.; Su, D.; Wang, W.; Chen, X.; Shao, Z. Fabrication of Air-Stable and Conductive Silk Fibroin Gels. ACS Appl. Mater. Interfaces 2018, 10, 38466-38475.

(27) Liu, L.; Han, Y.; Lv, S. Design of Self-Healing and Electrically Conductive Silk Fibroin-Based Hydrogels. ACS Appl. Mater. Interfaces 2019, 11, 20394-20403.

(28) Zhang, F.; Lu, Q.; Yue, X.; Zuo, B.; Qin, M.; Li, F.; Kaplan, D. L.; Zhang, X. Regeneration of High-quality Silk Fibroin Fiber by Wet 
Spinning from $\mathrm{CaCl}_{2}-$ Formic Acid Solvent. Acta Biomater. 2015, 12, $139-145$.

(29) Ling, S.; Zhang, Q.; Kaplan, D. L.; Omenetto, F.; Buehler, M. J.; Qin, Z. Printing of Stretchable Silk Membranes for Strain Measurements. Lab-on-a-Chip 2016, 16, 2459-2466.

(30) Jesorka, A.; Markström, M.; Karlsson, M.; Orwar, O. Controlled Hydrogel Formation in the Internal Compartment of Giant Unilamellar Vesicles. J. Phys. Chem. B 2005, 109, 14759-14763.

(31) Zhou, P.; Xie, X.; Knight, D. P.; Zong, X.-H.; Deng, F.; Yao, W.-H. Effects of $\mathrm{pH}$ and Calcium Ions on the Conformational Transitions in Silk Fibroin Using 2D Raman Correlation Spectroscopy and ${ }^{13} \mathrm{C}$ Solid-State NMR. Biochemistry 2004, 43, 11302-11311.

(32) Yu, C.; Wang, C.; Liu, X.; Jia, X.; Naficy, S.; Shu, K.; Forsyth, M.; Wallace, G. G. A Cytocompatible Robust Hybrid Conducting Polymer Hydrogel for Use in a Magnesium Battery. Adv. Mater. 2016, 28, 9349-9355.

(33) Chen, G.; Matsuhisa, N.; Liu, Z.; Qi, D.; Cai, P.j Jiang, Y.; Wan, C.; Cui, Y.; Leow, W. R.; Liu, Z.; et al. Plasticizing Silk Protein for On-Skin Stretchable Electrodes. Adv. Mater. 2018, 30, No. 1800129.

(34) Ghosh, S.; Inganäs, O. Self-assembly of a Conducting Polymer Nanostructure by Physical Crosslinking: Applications to Conducting Blends and Modified Electrodes. Synth. Met. 1999, 101, 413-416.

(35) Kim, D.; Park, K. Swelling and Mechanical Properties of Superporous Hydrogels of Poly(acrylamide-co-acrylic acid)/Polyethylenimine Interpenetrating Polymer Networks. Polymer 2004, 45, 189-196.

(36) Park, S. J.; Lee, K. Y.; Ha, W. S.; Park, S. Y. Structural Changes and Their Effect on Mechanical Properties of Silk Fibroin/Chitosan Blends. J. Appl. Polym. Sci. 1999, 74, 2571-2575.

(37) Hirst, A. R.; Escuder, B.; Miravet, J. F.; Smith, D. K. High-Tech Applications of Self-Assembling Supramolecular Nanostructured GelPhase Materials: From Regenerative Medicine to Electronic Devices. Angew. Chem., Int. Ed. 2008, 47, 8002-8018.

(38) Tee, B. C.; Wang, C.; Allen, R.; Bao, Z. An Electrically and Mechanically Self-healing Composite with Pressure- and FlexionSensitive Properties for Electronic Skin Applications. Nat. Nanotechnol. 2012, 7, 825-832.

(39) Garreau, S.; Louarn, G.; Buisson, J. P.; Froyer, G.; Lefrant, S. In Situ Spectroelectrochemical Raman Studies of Poly(3,4-ethylenedioxythiophene) (PEDT). Macromolecules 1999, 32, 6807-6812.

(40) Miserez, A.; Weaver, J. C.; Chaudhuri, O. Biological Materials and Molecular Biomimetics-Filling up the Empty Soft Materials Space for Tissue Engineering Applications. J. Mater. Chem. B 2015, 3, $13-24$.

(41) O’Leary, L. E. R.; Fallas, J. A.; Bakota, E. L.; Kang, M. K.; Hartgerink, J. D. Multi-Hierarchical Self-assembly of a Collagen Mimetic Peptide from Triple Helix to Nanofibre and Hydrogel. Nat. Chem. 2011, 3, 821-828.

(42) Huebsch, N.; Arany, P. R.; Mao, A. S.; Shvartsman, D.; Ali, O. A.; Bencherif, S. A.; Rivera-Feliciano, J.; Mooney, D. J. Harnessing Traction-Mediated Manipulation of the Cell/Matrix Interface to Control Stem-Cell Fate. Nat. Mater. 2010, 9, 518-526.

(43) Wang, Z.; Tammela, P.; Huo, J.; Zhang, P.; Strømme, M.; Nyholm, L. Solution-Processed Poly(3,4-ethylenedioxythiophene) Nanocomposite Paper Electrodes for High-capacitance Flexible Supercapacitors. J. Mater. Chem. A 2016, 4, 1714-1722.

(44) Yao, B.; Wang, H.; Zhou, Q.; Wu, M.; Zhang, M.; Li, C.; Shi, G. Ultrahigh-Conductivity Polymer Hydrogels with Arbitrary Structures. Adv. Mater. 2017, 29, No. 1700974.

(45) Li, W.; Gao, F.; Wang, X.; Zhang, N.; Ma, M. Strong and Robust Polyaniline-Based Supramolecular Hydrogels for Flexible Supercapacitors. Angew. Chem. 2016, 128, 9342-9347.

(46) Yang, H.; Xu, H.; Li, M.; Zhang, L.; Huang, Y.; Hu, X. Assembly of $\mathrm{NiO} / \mathrm{Ni}(\mathrm{OH})_{2} /$ PEDOT Nanocomposites on Contra Wires for Fiber-Shaped Flexible Asymmetric Supercapacitors. ACS Appl. Mater. Interfaces 2016, 8, 1774-1779. 